\title{
Reduced macular vessel density in COVID-19 patients with and without associated thrombotic events using optical coherence tomography angiography
}

\author{
Noemi Guemes-Villahoz ${ }^{1}$ B Barbara Burgos-Blasco ${ }^{1}$ - Beatriz Vidal-Villegas ${ }^{1}$. Juan Donate-López ${ }^{2}$. \\ María Herrera de la Muela ${ }^{3} \cdot$ Lorenzo López-Guajardo $^{2} \cdot$ Francisco Javier Martín-Sánchez $^{4} \cdot$ Julián García-Feijoó $^{5}$
}

Received: 20 November 2020 / Revised: 15 March 2021 / Accepted: 7 April 2021 / Published online: 7 May 2021

(c) The Author(s), under exclusive licence to Springer-Verlag GmbH Germany, part of Springer Nature 2021

\begin{abstract}
Purpose Thrombotic events (TE) represent one of the major complications of SARS-CoV-2 infection. The objective is to evaluate vessel density (VD) and perfusion density (PD) by optical coherence tomography angiography (OCTA) in COVID19 patients, and compare the findings with healthy controls. The secondary objective is to evaluate if there are differences in OCTA parameters between COVID-19 patients with and without associated TE.

Methods Cross-sectional case-control study that included patients with laboratory-confirmed diagnosis of COVID-19 with and without TE related to the infection and age-matched healthy controls. Ophthalmological examination and OCTA were performed 12 weeks after diagnosis. Demographic data and medical history were collected. Macular OCTA parameters in the superficial retinal plexus were analyzed according to ETDRS sectors.

Results Ninety patients were included, 19 (20\%) COVID-19 patients with associated TE, 47 (49.5\%) COVID-19 patients without TE, and 29 (30.5\%) healthy controls. Fifty-three (55.7\%) were male, mean age 54.4 (SD 10.2) years. COVID19 patients presented significantly lower VD than healthy controls: central $(p=0.003)$, inner ring $(p=0.026)$, outer ring $(p=0.001)$. PD was also significantly decreased: outer ring $(p=0.003)$, full area $(p=0.001)$. No differences in OCTA parameters were found between COVID-19 patients with and without TE.

Conclusions OCTA represents a promising tool for the in vivo assessment of microvascular changes in COVID-19. Patients with SARS-CoV-2 infection show lower VD and PD compared to healthy controls. However, no differences were found between COVID-19 when considering TE. Prospective studies are required to further evaluate the retinal microvascular involvement of SARS-CoV-2 and its impact on the vasculature of other organs.
\end{abstract}

Noemi Guemes-Villahoz

noemiguemes@gmail.com

1 Ophthalmology Department, Hospital Clínico San Carlos, Instituto de Investigación Sanitaria del Hospital Clínico San Carlos (IdISSC), Madrid, Spain

2 Ophthalmology Department, Hospital Clínico San Carlos, Instituto de Investigación Sanitaria del Hospital Clínico San Carlos (IdISSC), Oftared, Madrid, Spain

3 Obstetrics and Gynecology Department, Instituto de Salud de La Mujer, Instituto de Investigación Sanitaria del Hospital Clínico San Carlos (IsISSC), Madrid, Spain
4 Department of Emergency, Hospital Clinico San Carlos, Instituto de Investigación Sanitaria del Hospital Clínico San Carlos (IdISSC), Madrid, Spain

5 Ophthalmology Department, Hospital Clínico San Carlos, Departamento de Inmunología, Oftalmología y ORL,

Facultad de Medicina, Universidad Complutense de Madrid, Instituto de Investigación Sanitaria del Hospital Clínico San Carlos (IdISSC), Oftared, Madrid, Spain 


\section{Key messages}

- Optical coherence angiography (OCTA) represents a promising tool in the in vivo study of microvascular involvement in COVID-19 patients.

- Patients with SARS-CoV-2 infection show reduced vessel density and perfusion density compared to healthy controls.

- OCTA parameters showed no difference between COVID-19 patients when considering the history of COVID-related thrombotic events.

- Retinal vasculature imaging with OCTA may provide a potential biomarker of vascular changes related to SARS-CoV-2 in other organs.

Keywords COVID-19 $\cdot$ SARS-CoV-2 $\cdot$ OCTA $\cdot$ Retina $\cdot$ Thrombotic events

\section{Introduction}

Coronavirus disease (COVID-19) caused by severe acute respiratory syndrome coronavirus 2 (SARS-CoV-2) has reached pandemic proportions in an unprecedented time frame. Since its emergence, a number of associated complications have been described that may occur during both the acute and late phases of the disease. Among these complications, the development of thrombotic events (TE), such as pulmonary embolism (PE) and deep vein thrombosis (DVT), has been described [1, 2]. The actual mechanisms of COVID-19-induced thrombosis have not been elucidated, although it is speculated that a causal and bidirectional relationship between inflammation and thrombosis may be the origin of these phenomena [3].

Optical coherence tomography angiography (OCTA) is a novel and non-invasive technique that generates a three-dimensional angiogram of the retina, allowing a quantitative evaluation of retinal blood vessels without the need for intravenous contrast [4], which makes it a promising technique in the study of microangiopathies and thrombotic phenomena related to COVID-19. Compared to qualitative evaluations, quantitative objective measurements of retinal vascularization offer the possibility of early and accurate detection of subtle microvascular abnormalities not clinically detectable.

Since retinal vascularization shares morphological and pathophysiological characteristics with the vasculature of other organs, the study of retinal microvasculature by OCTA has been used to evaluate other systemic diseases, including cardiovascular and infectious diseases [5-7]. Therefore, the assessment of retinal vascularization in COVID-19 patients is of considerable value, especially in those who have suffered vascular complications associated with the infection. To the best of our knowledge, no studies evaluating retinal circulation by OCTA in COVID-19 patients who developed TE related to the disease have been published to date.

The aim of this study is to evaluate the vessel density and perfusion density by OCTA in COVID-19 patients, and to compare the findings with healthy controls. The secondary objective is to evaluate if there are differences in OCTA parameters between COVID-19 patients when considering the history of TE.

\section{Methods}

\section{Subjects}

Cross-sectional case-control study was conducted at the Hospital Clinico San Carlos (HCSC) in Madrid, Spain. The study was approved by the HCSC's Clinical Research Ethics Committee and was conducted in accordance with the Declaration of Helsinki. Written informed consent was obtained from all patients.

The study selected 3 groups of patients: patients with confirmed diagnosis of COVID-19 who presented TE associated to the infection (group 1), patients with confirmed diagnosis of COVID-19 without TE (group 2), and healthy controls (group 3). The groups were matched by age, sex, and refraction.

For group 1, patients with laboratory-confirmed diagnosis of COVID-19 who presented TE associated with the disease were selected from a cohort of patients attended at HCSC from March 23 to May 31, 2020. The inclusion criteria for group 1 were as follows: age 18 to 70 years, positive reverse transcriptase polymerase chain reaction (RT-PCR) test from nasopharyngeal swab for SARS-CoV-2, TE presented during the illness in the absence of any disease, or coagulation disorder that might justify it. The TE considered 
were pulmonary embolism (PE) in a patient with COVID-19 pneumonia confirmed by computed tomography angiography and/or deep vein thrombosis confirmed by ultrasound. The criteria also included blood tests performed during hospital admission and written informed consent for study participation. Critically ill patients requiring admission to the intensive care unit (ICU), those with concomitant eye diseases, quarantined patients, those unable to attend to the hospital, or lacking consent to participate in the study were excluded.

Group 2 included patients who attended the emergency department (ED) of HCSC from March 23 to 26, 2020, and presented a laboratory-confirmed diagnosis of SARS-CoV-2 infection. The inclusion and exclusion criteria for this group were the same as for group 1, except for the presence of TE related to COVID-19.

The control group (group 3) included a cohort of healthy patients who attended the ophthalmology department for routine eye examinations. Inclusion criteria for this group were as follows: age 18 to 70 years, negative laboratory tests for SARS-CoV-2 infection (IgG and IgMELISA serology and PCR from nasopharyngeal swab, both negative), absence of symptoms compatible with COVID19 or close contact with COVID-19 patient in the 14 days prior to the evaluation, absence of concomitant eye diseases, and written informed consent for participation in the study.

Eye diseases excluded for all three groups were high myopia ( $>6$ diopters), retinal vascular disease, macular and optic nerve disease, previous ocular surgery other than uncomplicated cataract surgery performed at least 6 months prior to the evaluation, and media opacity affecting OCTA's scan or image quality.

Demographic data, medical history (arterial hypertension, diabetes mellitus, dyslipidemia, obesity, chronic respiratory disease and cardiac disease, smoking), clinical evaluation data (onset of symptoms, oxygen saturation upon arrival, consolidation on chest X-ray, and clinical severity according to the WHO ordinal scale) [8], and laboratory test results (complete hemogram, lipid profile, C-reactive protein (PCR), troponins, ferritin, fibrinogen, lactate dehydrogenase levels (LDH), and D-dimer (DD)) during the hospital stay were collected. As for the laboratory tests, the highest levels documented during the hospital stay were considered.

\section{Ophthalmic examination}

Eye exam included a slit lamp examination of the anterior segment, a fundus examination and OCTA 12 weeks after the diagnosis of the infection. All procedures performed followed infection prevention and control measures according to hospital's protocol.

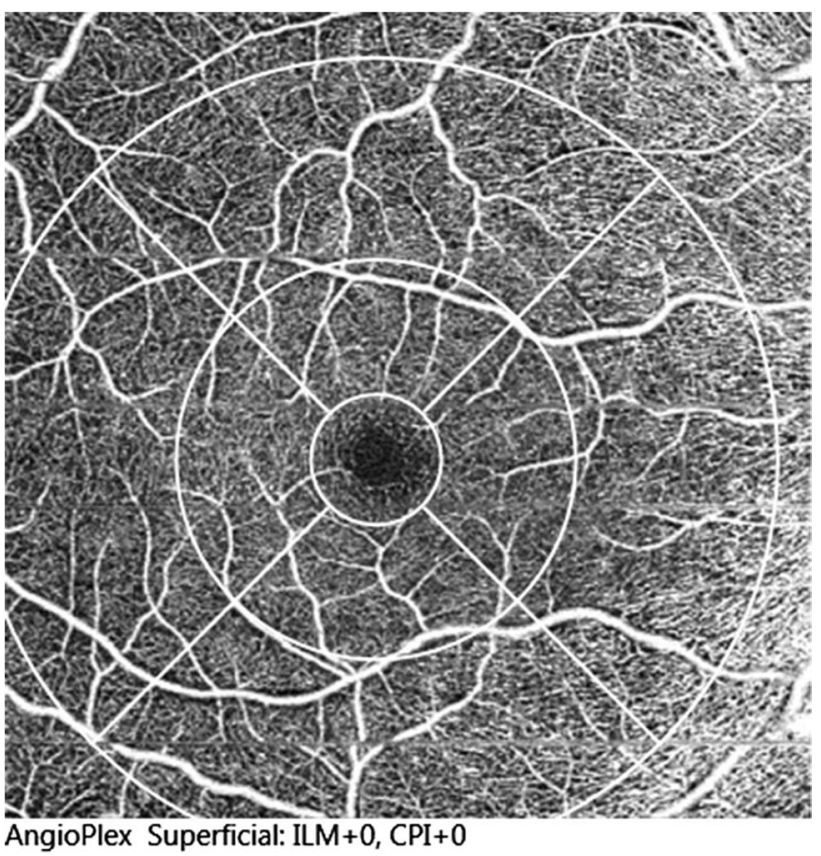

Fig. 1 OCTA of the macular region in the superficial capillary plexus, depicting the 9 sectors of the ETDRS grid. Central circle and inner and outer rings, which are further subdivided into superior, nasal, inferior and temporal regions

OCTA images were obtained using the Zeiss Cirrus 5000 spectral domain OCTA with AngioPlex (Carl Zeiss Meditec, Inc., Dublin, CA, USA). Macular angiography images were performed using the $6 \times 6 \mathrm{~mm}$ macular cube protocol in both eyes for each subject. The inclusion criteria for acceptable signal strength (SS) was 7 or more. The complex optical microangiography (cOMAG) algorithm analyzed changes in the complex signals. The results were analyzed using Cirrus OCTA software (AngioPlex ${ }^{\mathrm{TM}}$, version 11.0).

Eye exam was performed by two ophthalmologists (NG and BB). OCTA was performed by the same examiner, and reviewed individually by the same two ophthalmologists for quality assessment, excluding those images with lower quality. OCTA data of the superficial capillary plexus (SCP) were collected, including vessel density (VD), perfusion density (PD), and the area, perimeter, and morphology (circularity) of the foveal avascular zone (FAZ). VD was defined as the total length of perfused vasculature per unit area in the region of measurement and PD was defined as the total area of perfused vasculature per unit area in the same region. The built-in analytic algorithm automatically outlined the FAZ boundary along the innermost capillaries, quantifying the area and perimeter of this zone. FAZ circularity was defined as $4 \pi A / P$, where $A$ was the area and $P$ was the perimeter.

The macular region was segmented according to the nine sectors of the Study of Early Treatment of Diabetic Retinopathy (ETDRS). The fovea was represented by a 


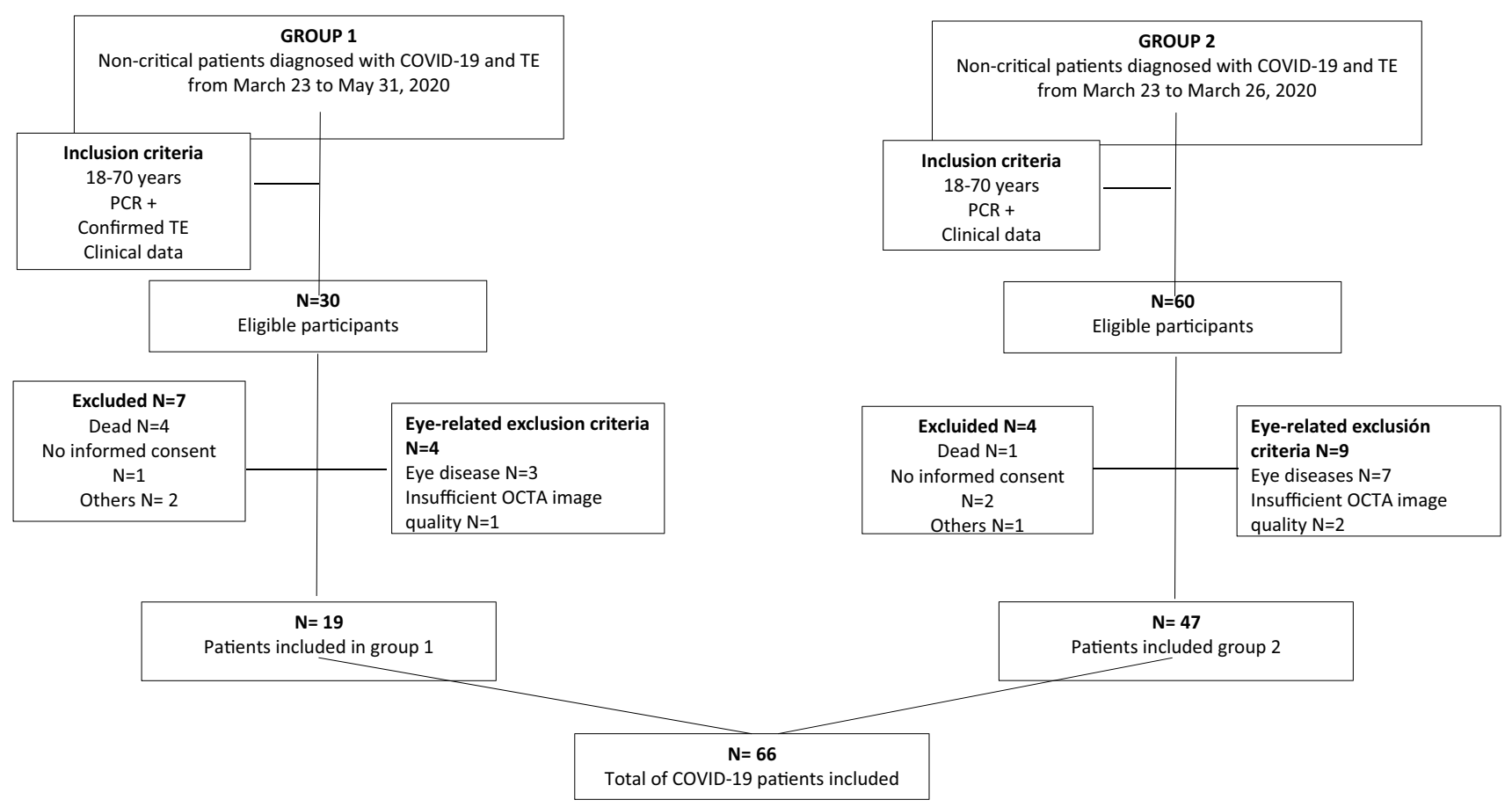

Fig. 2 Flowchart of COVID-19 patients included in the study

central circle of $1 \mathrm{~mm}$ in diameter. The inner ring had an inner diameter of $1 \mathrm{~mm}$ and an outer diameter of $3 \mathrm{~mm}$, and the outer circle had an inner diameter of $3 \mathrm{~mm}$ and an outer diameter of $6 \mathrm{~mm}$ (Fig. 1). Both inner and outer rings were further subdivided into upper, nasal, lower, and temporal regions. The subjects' right eye was included, unless it did not meet the criteria for inclusion and exclusion, in which case the left eye was included.

\section{Statistics}

All statistical tests were performed using the software package IBM SPSS (version 25.0; IBM Corp., Somers, NY, USA). Demographic and clinical data of the COVID19 patients is presented as mean and standard deviation for quantitative variables, while prevalence of categorical variables will be presented as percentage. Differences in age and
Table 1 Clinical characteristics of COVID-19 patients

\begin{tabular}{llll}
\hline & $\begin{array}{l}\text { Group 1 } \\
\text { COVID-19 with TE } \\
(n=19)\end{array}$ & $\begin{array}{l}\text { Group 2 } \\
\text { COVID-19 without TE } \\
(n=47)\end{array}$ & $p$ \\
\hline Demographic data & & & \\
Age (mean [SD] $)$ & $57.1(6.0)$ & $57.3(8.2)$ & 0.260 \\
Male $(n[\%])$ & $13(68.4)$ & $27(57.4)$ & 0.260 \\
Female $(n[\%])$ & $6(31.6)$ & $20(42.6)$ & 0.603 \\
Medical history & & & 0.224 \\
Arterial hypertension $(n[\%])$ & $4(25.0)$ & $15(31.9)$ & 0.271 \\
Diabetes mellitus $(n[\%])$ & $5(31.2)$ & $15(17.0)$ & 0.773 \\
Dyslipidemia $(n[\%])$ & $4(25.0)$ & $4(8.5)$ & 0.556 \\
Cardiac disease $(n[\%])$ & $1(6.2)$ & $1(2.1)$ & 0.092 \\
Renal disease $(n[\%])$ & $0(0)$ & $1(2.1)$ & 0.434 \\
Liver disease $(n[\%])$ & $2(12.5)$ & $3(6.4)$ & 0.985 \\
Cancer $(n[\%])$ & $2(12.5)$ & $3(6.4)$ & \\
Chronic respiratory disease $(n[\%])$ & $1(6.2)$ & & \\
\hline
\end{tabular}


sex between COVID-19 patients and the control group were compared using the Mann-Whitney $U$ test and the chi ${ }^{2}$ test.

The OCT data of both groups are represented as means and standard deviation. The normality of the variables was confirmed with the Kolmogorov-Smirnov test. To analyze the differences in the OCT parameters between COVID-19 patients and healthy controls, a $t$-student test was performed. Likewise, to compare the group of patients with and without TE, a $t$-student test was performed. Statistical significance was set at $p<0.05$.

\section{Results}

The total study population included 95 patients, 66 (69.4\%) COVID-19 patients, and 29 (30.5\%) healthy controls. Among the 66 COVID-19 patients, 19 (28.8\%) belonged to group 1 and $47(71.2 \%)$ to group 2 . Of the non-critically ill patients diagnosed with COVID-19 and TE from March 23 to May 31, 2020, 30 patients met the inclusion criteria. Among these, 19 patients were finally included in group 1 . All of them (19/19) presented PE, and $2(2 / 19)$ presented both PE and DVT related to COVID-19. Likewise, of the non-critical COVID-19 patients that attended ED on the dates indicated, 47 patients met the inclusion and exclusion criteria (group 2). Figure 2 shows the flow chart of the cases included in the study.

Demographic and clinical characteristics of COVID-19 patients are summarized in Table 1 . The mean age of healthy controls was 51.0 (SD 11.9). There were no statistically significant differences between groups in terms of age and sex.

Eye exam was performed 88 days (86-90) after COVID19 diagnosis and revealed the ocular diseases previously

Table 2 OCTA data in COVID-19 patients and healthy controls

\begin{tabular}{|c|c|c|c|c|c|}
\hline \multirow[t]{2}{*}{ Variable } & \multicolumn{2}{|c|}{ COVID-19 $(N=66)$} & \multicolumn{2}{|c|}{$\begin{array}{l}\text { Healthy controls } \\
(N=29)\end{array}$} & \multirow[t]{2}{*}{$p$} \\
\hline & Mean & SD & Mean & SD & \\
\hline VD central & 7.77 & 3.26 & 10.10 & 3.41 & 0.003 \\
\hline VD inner & 16.49 & 2.47 & 27.52 & 1.82 & 0.026 \\
\hline VD outer & 17.28 & 2.15 & 18.48 & 1.21 & 0.001 \\
\hline VD complete & 16.89 & 2.02 & 17.72 & 2.49 & 0.118 \\
\hline PD central & 20.05 & 25.35 & 22.41 & 7.84 & 0.494 \\
\hline PD inner & 39.18 & 6.76 & 56.17 & 75.30 & 0.235 \\
\hline PD outer & 42.94 & 5.65 & 45.79 & 3.22 & 0.003 \\
\hline PD complete & 41.46 & 4.99 & 44.35 & 3.30 & 0.001 \\
\hline FAZ a & 0.50 & 1.87 & 0.12 & 0.44 & 0.152 \\
\hline FAZ p & 2.10 & 0.62 & 2.28 & 0.68 & 0.262 \\
\hline FAZ c & 0.68 & 0.11 & 1.96 & 3.81 & 0.106 \\
\hline
\end{tabular}

The significance of the $p$ values is in bold $(p<0.05)$

$S D$, standard deviation; $V D$, vessel density; $P D$, perfusion density; $F A Z$, foveal avascular zone; $a$, area; $p$, perimeter; $c$, circularity defined as exclusion criteria in a total of 10 patients, who were excluded from the study. Fundus examination of the 66 patients with a history of SARS-CoV-2 infection was unremarkable. No retinal vascular changes, macular or optic nerve involvement, were evident in any of the groups. Neither did any patient report decreased vision or other noticeable ocular symptoms during the disease, nor until the time of the evaluation.

Significant differences in VD and PD were detected by OCTA between COVID-19 patients and healthy controls. COVID-19 patients presented significantly lower VD than healthy controls: central $(p=0.003)$, inner ring $(p=0.026)$, outer ring $(p=0.001)$. PD was also decreased in COVID19 patients: outer ring $(p=0.003)$, full area $(p=0.001)$. Table 2 shows macular OCTA data of COVID-19 patients and healthy controls. (Additional information is provided in a supplementary table). However, within the COVID-19 group, no differences in OCTA parameters were found when considering history of TE.

Laboratory parameters evidenced significant differences in D-dimer levels between the group with and without TE $(p=0.015)$. Group 1 revealed mean D-dimer values of 5528.5 (SD 6204.3) and group 2 of 648.2 (SD 401.5).

\section{Discussion}

Thrombotic events are emerging as one of the most relevant sequelae of SARS-CoV-2 infection [9, 10]. A high incidence of TE has been described in COVID-19 patients, more so in critically ill patients, reporting variable incidences ranging from 11.5 to $27 \%$ [1, 10-14]. Although PE represents the most frequent thrombotic manifestation associated with the infection [1], TE, venous and arterials, have been also described in other organs such as the eye [15]. Our study reveals quantitative differences in retinal microcirculation between COVID-19 patients and healthy controls. Patients with history of SARS-CoV-2 infection had significantly lower vessel density and perfusion density than healthy controls 3 months after the acute phase of the infection. These findings provide relevant information about the microvascular involvement of SARS-CoV-2 infection and the possible vascular sequelae of COVID-19. Nevertheless, no differences in these parameters have been detected in COVID19 patients when considering the history of TE, which may indicate that the microvascular involvement of SARS-CoV-2 at the retinal level is not conditioned by the presence of TE at other levels. Similarly, no increased incidence of retinal vascular events has been found in COVID-19 patients who had TE, compared to those who did not.

OCTA is a novel technique that allows the study and objective quantification of retinal vasculature, assessing the retinal microvascular impact of the disease. The fact that the 
VD and PD are significantly reduced in COVID-19 patients even 3 months after the acute phase of the infection exhibits important questions about the mechanism of microvascular damage of SARS-CoV-2 and its possible mid- and longterm sequels, both in the eye and other organs. To date, the mechanism by which SARS-CoV-2 may affect the retina vasculature is unknown. Recently, SARS-CoV-2 RNA has been detected in retinal biopsies performed postmortem in patients with COVID-19 [16]. The detection of the virus in intraocular tissue could be explained by the presence of angiotensin-converting enzyme 2 (ACE2), the main receptor of SARS-CoV-2 cellular entry, in the retina [17]. In this sense, the presence of ACE2 has also been detected in arterial and venous endothelial cells [18]. It then seems feasible that vascular changes may occur at this level.

Furthermore, thrombosis and extrapulmonary manifestations have been reported without confirmed virus presence at these sites [19], suggesting that SARS-CoV-2 may cause both direct cytopathic damage and indirect damage related to the intense inflammatory response and hypercoagulable state it induces. These findings have yielded to the concept of thrombo-inflammation as key phenomena in the pathophysiology of COVID-19, whereby inflammation activates coagulation, and coagulation heightens inflammatory activity [20, 21]. This rationale is consistent with the findings reported in autopsies of COVID-19 patients who have died from the disease, which have revealed microvasculature thrombi as prominent feature in multiple organs [22]. All these COVID-19-related vascular phenomena might partly explain the findings encountered in our study.

The post-mortem examination for further evaluation of the vascular damage in the lungs and other organs is supported. However, retinal circulation has the unique advantage of being easily studied in vivo. Hence, OCTA may provide a valuable tool in the study of microvascular involvement in COVID-19 patients, since it may represent a potential biomarker of vascular damage in other organs $[5,23,24]$.

Current evidence about the presence of retinal venous thrombosis in COVID-19 patients is scarce. In this sense, it has been reported a case of a 52-year-old male without cardiovascular risk factors, and normal coagulation study who presented a branch retinal vein occlusion 10 days after the onset of fever and malaise with subsequent laboratory confirmation of SARS-CoV-2 infection [15]. Another case of a 54-year-old woman with COVID-19-associated pneumonia and impending retinal vein occlusion has also been recently described. She presented decreased visual acuity and retinal changes compatible with impending central retinal vein occlusion, which resolved after treatment and resolution of the pneumonia [25]. All this suggests that the hypercoagulability state associated with COVID-19 may also clinically affect retinal vasculature.
Our study has several limitations. First, this study did not include critically ill patients requiring ICU admission. Since the incidence of TE in critical COVID-19 patients is much higher, clinical and subclinical findings might appear more evident in this group of patients. In addition, critically ill patients present progressive and sustained hypoxia that has been associated with an exaggerated release of proinflammatory and prothrombotic factors [26, 27], which could aggravate the changes found in retinal microcirculation. On the other hand, the eye exam was performed 12 weeks after COVID-19 diagnosis, so certain retinal vascular changes that might occur during the acute phase of the infection could not be clinically present in the subsequent examination. Although it would have been interesting to evaluate patients during the acute phase of infection, the extremely critical scenario and hospital logistics prevented such evaluation during the peak of the pandemic. Finally, the number of patients recruited in the study is limited. Prospective studies with larger numbers of patients may yield more data about the microvascular impact of SARS-CoV-2 infection.

In conclusion, the evaluation of retinal circulation represents a promising tool in the in vivo study of microvascular involvement in COVID-19 patients. Patients with SARSCoV-2 infection show decreased retinal vessel density and perfusion density compared to healthy controls as late as 12 weeks after the acute phase of infection. However, no differences in OCTA parameters have been found between COVID-19 patients when considering the history of TE. These findings provide relevant information about the microvascular damage of SARS-CoV-2, even in the absence of TE, and its possible impact on the vasculature of other organs.

Supplementary Information The online version contains supplementary material available at https://doi.org/10.1007/s00417-021-05186-0.

Acknowledgements We wish to thank participating investigators that helped collecting data: Ana González Álvarez-Nava, Helga Tallon Ávila, María Isabel Sánchez Perea, María Carmen Rivera Sequera. COVID-19_URG-HCSC Register: Juan González del Castillo, Adrián Valls Carbó, Enrique del Toro, Eduardo Cardassay, Gabriel Cozar López, María del Mar Suárez-Cadenas, Pablo Jerez Fernández, Beatriz Angós, Cristina Díaz del Arco, Esther Rodríguez Adrada, María Teresa Montalvo Moraleda, Carolina Espejo Paeres, Amanda López Picado, Carmen Martínez Valero, Juande D. Miranda, David Chaparro, Miguel Ángel García Briñón, José Luis Fernández Rueda, José M ${ }^{\mathrm{a}}$ Leal Pozuelo, José Luis Fernández Rueda, Víctor Hernández Martín-Romo.

Author contribution All named authors meet the International Committee of Medical Journal Editors (ICMJE) criteria for authorship for this article and take responsibility for the integrity of the work as a whole.

Data Availability Data sharing is not applicable to this article as no datasets were generated or analyzed during the current study. 


\section{Declarations}

Ethics approval The study was approved by the Clinical Research Ethics Committee of HCSC and was conducted in accordance with the Helsinki Declaration.

Consent to participate Written informed consent was obtained from all patients.

Consent for publication All authors have given their approval for this version to be published.

Conflict of interest The authors declare no competing interests.

\section{References}

1. Llitjos J-F, Leclerc M, Chochois C et al (2020) High incidence of venous thromboembolic events in anticoagulated severe COVID19 patients. J Thromb Haemost 18:1743-1746. https://doi.org/10. 1111/jth. 14869

2. Demelo-Rodríguez P, Cervilla-Muñoz E, Ordieres-Ortega L et al (2020) Incidence of asymptomatic deep vein thrombosis in patients with COVID-19 pneumonia and elevated D-dimer levels. Thromb Res 192:23-26. https://doi.org/10.1016/j.thromres.2020. 05.018

3. Abou-Ismail MY, Diamond A, Kapoor S et al (2020) The hypercoagulable state in COVID-19: incidence, pathophysiology, and management. Thromb Res 194:101-115. https://doi.org/10.1016/j. thromres.2020.06.029

4. Hagag A, Gao S, Jia Y, Huang D (2017) Optical coherence tomography angiography: technical principles and clinical applications in ophthalmology. Taiwan J Ophthalmol 7:115. https://doi.org/10. 4103/tjo.tjo_31_17

5. Pascual-Prieto J, Burgos-Blasco B, Ávila Sánchez-Torija M et al (2020) Utility of optical coherence tomography angiography in detecting vascular retinal damage caused by arterial hypertension. Eur J Ophthalmol 30:579-585. https://doi.org/10.1177/11206 72119831159

6. Akmaz B, Akay F, Güven YZ et al (2020) The long-term effect of human immunodeficiency virus infection on retinal microvasculature and the ganglion cell-inner plexiform layer: an OCT angiography study. Graefes Arch Clin Exp Ophthalmol 258:1671-1676. https://doi.org/10.1007/s00417-020-04749-x

7. Patton N, Aslam T, Macgillivray T et al (2005) Retinal vascular image analysis as a potential screening tool for cerebrovascular disease: a rationale based on homology between cerebral and retinal microvasculatures. J Anat 206:319-348. https://doi.org/ 10.1111/j.1469-7580.2005.00395.x

8. WHO. Ordinal Scale COVID-19. https://www.who.int/bluep rint/priority-diseases/key-action/COVID-19_Treatment_ Trial_Design_Master_Protocol_synopsis_Final_18022020. pdf. Accessed 24 Oct 2020

9. Miró Ò, Llorens P, Aguirre A et al (2020) Association between Covid-19 and pulmonary embolism (AC-19-PE study). Thromb Res 196:322-324. https://doi.org/10.1016/j.thromres.2020.09.010

10. Benito N, Filella D, Mateo J et al (2020) Pulmonary Thrombosis or embolism in a large cohort of hospitalized patients with Covid19. Front Med 7:557. https://doi.org/10.3389/fmed.2020.00557

11. Rali P, O'Corragain O, Oresanya L, et al (2020) Incidence of venous thromboembolism (VTE) in COVID-19: an experience from a single large academic center. J Vasc Surg Venous Lymphat Disord. https://doi.org/10.1016/j.jvsv.2020.09.006
12. Al-Ani F, Chehade S, Lazo-Langner A (2020) Thrombosis risk associated with COVID-19 infection A scoping review. Thromb Res 192:152-160. https://doi.org/10.1016/j.thromres.2020.05.039

13. Griffin DO, Jensen A, Khan M et al (2020) Pulmonary embolism and increased levels of d-dimer in patients with coronavirus disease. Emerg Infect Dis 26:1941-1943. https://doi.org/10.3201/ eid2608.201477

14. Giorgi-Pierfranceschi M, Paoletti O, Pan A, et al (2020) Prevalence of asymptomatic deep vein thrombosis in patients hospitalized with SARS-CoV-2 pneumonia: a cross-sectional study. Intern Emerg Med. https://doi.org/10.1007/s11739-020-02472-3

15. Sheth JU, Narayanan R, Goyal J, Goyal V (2020) Retinal vein occlusion in COVID-19: a novel entity. Indian J Ophthalmol 68:2291-2293. https://doi.org/10.4103/ijo.IJO_2380_20

16. Casagrande M, Fitzek A, Püschel K et al (2020) Detection of SARS-CoV-2 in human retinal biopsies of deceased COVID-19 Patients. Ocul Immunol Inflamm 28:721-725. https://doi.org/10. 1080/09273948.2020.1770301

17. deS Senanayake P, Drazba J, Shadrach K, et al (2007) Angiotensin II and its receptor subtypes in the human retina. Invest Ophthalmol Vis Sci 48:3301-11. https://doi.org/10.1167/iovs.06-1024

18. Hamming I, Timens W, Bulthuis MLC et al (2004) Tissue distribution of ACE2 protein, the functional receptor for SARS coronavirus. A first step in understanding SARS pathogenesis. J Pathol 203:631-637. https://doi.org/10.1002/path.1570

19. Sekhawat V, Green A, Mahadeva U (2021) COVID-19 autopsies: conclusions from international studies. Diagn Histopathol (Oxf) 27:103-107. https://doi.org/10.1016/j.mpdhp.2020.11.008

20. Levi M, van der Poll T, Büller HR (2004) Bidirectional relation between inflammation and coagulation. Circulation 109:26982704. https://doi.org/10.1161/01.CIR.0000131660.51520.9A

21. Gris J-C, Perez-Martin A, Quéré I, Sotto A (2020) COVID-19 associated coagulopathy: the crowning glory of thrombo-inflammation concept. Anaesthesia Crit Care Pain Med 39:381-382. https://doi.org/10.1016/j.accpm.2020.04.013

22. Carsana L, Sonzogni A, Nasr A et al (2020) Pulmonary post-mortem findings in a series of COVID-19 cases from northern Italy: a two-centre descriptive study. Lancet Infect Dis 20:1135-1140. https://doi.org/10.1016/S1473-3099(20)30434-5

23. Lee C-W, Cheng H-C, Chang F-C, Wang A-G (2019) Optical coherence tomography angiography evaluation of retinal microvasculature before and after carotid angioplasty and stenting. Sci Rep 9:14755. https://doi.org/10.1038/s41598-019-51382-8

24. Lee J-Y, Kim JP, Jang H et al (2020) Optical coherence tomography angiography as a potential screening tool for cerebral small vessel diseases. Alzheimers Res Ther 12:73. https://doi.org/10. 1186/s13195-020-00638-x

25. Invernizzi A, Pellegrini M, Messenio D, et al (2020) Impending central retinal vein occlusion in a patient with coronavirus disease 2019 (COVID-19). Ocul Immunol Inflamm 1-3. https://doi.org/ $10.1080 / 09273948.2020 .1807023$

26. Somers VK, Kara T, Xie J (2020) Progressive hypoxia. Mayo Clin Proc 95:2339-2342. https://doi.org/10.1016/j.mayocp.2020. 09.015

27. Kashani KB (2020) Hypoxia in COVID-19: Sign of severity or cause for poor outcomes. Mayo Clin Proc 95:1094-1096. https:// doi.org/10.1016/j.mayocp.2020.04.021

Publisher's note Springer Nature remains neutral with regard to jurisdictional claims in published maps and institutional affiliations. 